\title{
Pacific
}

Journal of

Mathematics

\section{IMBEDDING AND MULTIPLIER THEOREMS FOR DISCRETE}

\section{LITTLEWOOD-PALEY SPACES}

IGOR E. VERBITSKY

Volume $176 \quad$ No. 2

December 1996 


\title{
IMBEDDING AND MULTIPLIER THEOREMS FOR DISCRETE LITTLEWOOD-PALEY SPACES
}

\author{
IGOR E. VERBITSKY
}

We prove imbedding and multiplier theorems for discrete Littlewood-Paley spaces introduced by M. Frazier and B. Jawerth in their theory of wavelet-type decompositions of Triebel-Lizorkin spaces. The corresponding inequalities for discrete spaces defined in terms of characteristic functions of dyadic cubes, with respect to an arbitrary positive locally finite measure on the Euclidean space, are useful in the theory of tent spaces, weighted inequalities, duality theorems, interpolation by analytic and harmonic functions, etc. Our main tools are vector-valued maximal inequalities, a dyadic version of the Carleson measure theorem, and Pisier's factorization lemma. We also consider more general inequalities, with an arbitrary family of measurable functions in place of characteristic functions of dyadic cubes, which occur in the factorization theory of operators.

\section{Introduction.}

Let $\mathcal{Q}=\{Q\}$ be the family of all dyadic cubes in $\mathbf{R}^{n}$. Let $\omega$ be a nonnegative locally finite Borel measure on $\mathbf{R}^{n}$ such that $\int_{\partial Q} d \omega=0$ for all $Q \in \mathcal{Q}$. We set $\mathcal{Q}_{0}=\left\{Q \in \mathcal{Q}:|Q|_{\omega} \neq 0\right\}$, where $|Q|_{\omega}=\int_{Q} d \omega ;|Q|$ will stand for the Lebesgue measure of $Q$. For any $Q \in \mathcal{Q}_{0}$, we denote by $\widetilde{\chi}_{Q}$ its normalized characteristic function $\tilde{\chi}_{Q}=|Q|_{\omega}^{-1 / 2} \chi_{Q}$.

For $-\infty<\alpha<\infty, 0<p<\infty$, and $0<q \leq \infty$, we define the discrete Littlewood-Paley space $\mathbf{f}=\mathbf{f}_{p}^{\alpha q}(\omega)([\mathbf{7}],[8])$ as the space of sequences of reals, $s=\left\{s_{Q}\right\}_{Q \in \mathcal{Q}_{0}}$, such that

$$
\|s\|_{\mathbf{f}_{p}^{\alpha q}(\omega)}=\left\|\left(\sum_{Q \in \mathcal{Q}_{0}}\left(|Q|^{-\alpha / n}\left|s_{Q}\right| \tilde{\chi}_{Q}\right)^{q}\right)^{1 / q}\right\|_{L^{p}(d \omega)}<\infty .
$$

Note that we use the normalized characteristic functions $\tilde{\chi}_{Q}$ in the defi. nition of $\mathbf{f}$ spaces in order to have the duality relation $\left[\mathbf{f}_{p}^{\alpha q}(\omega)\right]^{*}=\mathbf{f}_{p^{\prime}}^{-\alpha q^{\prime}}(\omega)$ with the usual pairing $\langle s, t\rangle=\sum s_{Q} t_{Q}\left(s \in \mathbf{f}_{p}^{\alpha q}(\omega), t \in \mathbf{f}_{p^{\prime}}^{-\alpha q^{\prime}}\right)$, at least for 
$1<p<\infty$ and $1<q<\infty$. (Here $1 / p+1 / p^{\prime}=1$ and $1 / q+1 / q^{\prime}=1$.) We will also follow the convention that $q^{\prime}=\infty$ for $0<q \leq 1$. Then, as we will see, the duality relation holds for all $0<q \leq \infty$.

Our goal is to characterize both forward and reverse imbeddings of $\mathbf{f}$ spaces into classical $l^{r}$ spaces with weights. Let $w=\left\{w_{Q}\right\}_{Q \in \mathcal{Q}}, w_{Q} \geq 0$, be a fixed sequence of scalar weights. Then $l^{r}(w)(0<r \leq \infty)$ is the sequence space with quasi-norm

$$
\|s\|_{l^{r}(w)}=\left(\sum_{Q \in \mathcal{Q}}\left|s_{Q}\right|^{r} w_{Q}\right)^{1 / r} .
$$

We set $l^{r}=l^{r}(w)$ in the unweighted case $w_{Q} \equiv 1$. Let $\phi_{Q}=$ $|Q|^{-\alpha / n} w_{Q}^{-1 / r} \tilde{\chi}_{Q}$. (Note that $\phi_{Q}$ is a constant multiple of $\chi_{Q}$.) Let us assume that $w_{Q}=0$ if $|Q|_{\omega}=0$. Then clearly the imbedding $\mathbf{f}_{p}^{\alpha q}(\omega) \subset l^{r}(w)$ is valid if and only if the inequality

$$
\left\|\left(\sum_{Q}\left|s_{Q}\right|^{q} \phi_{Q}^{q}\right)^{1 / q}\right\|_{L^{p}(d \omega)} \geq C\left(\sum_{Q}\left|s_{Q}\right|^{r}\right)^{1 / r}
$$

holds, where the sums on both sides of (1.2) are taken over all $Q \in \mathcal{Q}_{1}=$ $\left\{Q \in \mathcal{Q}: w_{Q} \neq 0\right\}$.

In the same manner, the reverse imbedding $l^{r}(w) \subset \mathbf{f}_{p}^{\alpha q}(\omega)$ is equivalent to the inequality

$$
\left\|\left(\sum_{Q}\left|s_{Q}\right|^{q} \phi_{Q}^{q}\right)^{1 / q}\right\|_{L^{p}(d \omega)} \leq C\left(\sum_{Q}\left|s_{Q}\right|^{r}\right)^{1 / r} .
$$

(Here we assume that $|Q|_{\omega}=0$ if $w_{Q}=0$.)

Similar estimates, sometimes with some other functions in place of constant multiples of $\chi_{Q}$, appear in the theory of wavelet and atomic decompositions, tent spaces, weighted norm inequalities, interpolation by analytic and harmonic functions, Banach space geometry problems, operator theory, etc. (See $[\mathbf{1}],[\mathbf{2}],[\mathbf{4}],[\mathbf{7}]-[\mathbf{1 1}],[\mathbf{1 4}]-[\mathbf{1 9}],[\mathbf{2 1}]$.) Several special cases of (1.2) and (1.3), mostly for $d \omega=d x$, are known. For some values of the indices $p, q, r$ these inequalities are equivalent to duality theorems for $\mathbf{f}$ spaces, or dyadic versions of the Carleson measure theorem (see $[\mathbf{2}],[\mathbf{7}],[\mathbf{1 0}], .[\mathbf{1 7}]$, $[\mathbf{1 9}],[\mathbf{2 1}]$, and the discussion below). In certain difficult cases the proofs use "local maximal functions" [7], or "stopping time" arguments [14], [15].

Our approach is to consider (1.2) and (1.3) simultaneously, making use of certain duality relationship between them, for all $p, q, r>0$ and $\omega$. The main 
tools are a generalization of the Fefferman-Stein vector-valued maximal theorem [5], the dyadic Carleson measure theorem [19], and Pisier's lemma [18] proved in the context of the factorization theory of operators (see Theorem A below). Even in the known cases some of our proofs seem to be new and easier than the original ones. (Note that there is a gap in the proof given in [7] for the duality theorem for $\mathbf{f}_{p}^{\alpha q}$ in the case $1 \leq p<\infty$ and $0<q<1$. This theorem, which is used in [7] to obtain the corresponding duality result for the distribution spaces $F_{p}^{\alpha q}$, is equivalent to a characterization of (1.2) in the case $r=1$ and $d \omega=d x$. See Sec. 3 where we give a proof in a more general setting using Pisier's lemma and the results of [21].)

It is also of interest to look at the relations between $\mathbf{f}$ spaces and weighted $l^{r}$ spaces from a more general point of view. Let $I$ be an index set, and let $\left\{\phi_{i}\right\}_{i \in I}$ be a fixed family of non-negative measurable functions on a measure space $(X, d \omega)$. For the three indices, $0<p<\infty, 0<q \leq \infty$, and $0<r \leq \infty$, we consider the following inequalities,

$$
\left\|\left(\sum_{i \in I}\left|s_{i}\right|^{q} \phi_{i}^{q}\right)^{1 / q}\right\|_{L^{p}(d \omega)} \leq C\|s\|_{l^{r}},
$$

and

$$
\left\|\left(\sum_{i \in I}\left|s_{i}\right|^{q} \phi_{i}^{q}\right)^{1 / q}\right\|_{L^{p}(d \omega)} \geq C\|s\|_{l^{r}}
$$

for all $s=\left\{s_{i}\right\}_{i \in I}$ (with finitely many nonzero reals $s_{i}$ ).

It follows from (1.4) that

$$
\sup _{i \in I}\left\|\phi_{i}\right\|_{L^{p}(d \omega)}<\infty
$$

and similarly (1.5) implies

$$
\inf _{i \in I}\left\|\phi_{i}\right\|_{L^{p}(d \omega)}>0
$$

The converse is true for certain values of $p, q$ and $r$ (see Lemma 2 below), but generally the problem of characterizing (1.4) or (1.5) is known to be very difficult.

The following theorems of B. Maurey and G. Pisier treat the special cases $q=r$ of (1.2), and $q=r$ or $q=\infty$ of (1.3). We denote by $D(\omega)$ the set of all "densities" $F \in L^{1}(d \omega)$ such that $F \geq 0$ and $\int F d \omega \leq 1$. 
Theorem A. Let $0<p<r<\infty$ and $s=\left\{s_{i}\right\}_{i \in I}$.

(i) The inequality

$$
\left\|\left(\sum_{i \in I}\left|s_{i}\right|^{r} \phi_{i}^{r}\right)^{1 / r}\right\|_{L^{p}(d \omega)} \leq C\|s\|_{l^{r}}
$$

holds for all finitely supported $s=\left\{s_{i}\right\}$ if and only if there exists $F \in$ $D(\omega)$ such that

$$
\sup _{i \in I}\left\|F^{-1 / p} \phi_{i}\right\|_{L^{r}\left(d \omega_{0}\right)}<\infty
$$

where $d \omega_{0}=F d \omega$.

(ii) The inequality

$$
\left\|\sup _{i \in I}\left(\left|s_{i}\right| \phi_{i}\right)\right\|_{L^{p}(d \omega)} \leq C\|s\|_{l^{r}}
$$

holds if and only if there exists $F \in D(\omega)$, such that

$$
\sup _{i \in I}\left\|F^{-1 / p} \phi_{i}\right\|_{L^{r \infty}\left(d \omega_{0}\right)}<\infty
$$

where $L^{r \infty}\left(d \omega_{0}\right)$ is a weak $L^{r}$ space with respect to the measure $d \omega_{0}=$ $F d \omega$.

Theorem A plays a central role in the Nikishin-Stein-Maurey-Pisier theory of factorization of operators. It also has interesting applications to weighted norm inequalities (see $[\mathbf{9}, \mathbf{2 1}]$ ). Statement (i) of Theorem A is proved in [16]; statement (ii) is obtained in [18], where also a different proof of (i) is given. It can be shown that, if $p<r$, then (1.4) actually does not depend on $q$ for $r<q \leq \infty$. Hence the "weak type" condition (1.9) characterizes (1.4) in this case. The following theorem is also proved in [16].

Theorem B. Let $0<r<p<\infty$. The inequality

$$
\left\|\left(\sum_{i \in I}\left|s_{i}\right|^{r} \phi_{i}^{r}\right)^{1 / r}\right\|_{L^{p}(d \omega)} \geq C\|s\|_{l^{r}}
$$

holds if and only there exists $F \in D(\omega)$, such that

$$
\inf _{i \in I}\left\|F^{-1 / p} \phi_{i}\right\|_{L^{r}\left(d \omega_{0}\right)}>0
$$


where $d \omega_{0}=F d \omega$.

It seems to be no general duality relations known for the inequalities (1.4) and (1.5). We will show below (Sec. 2) that actually Theorem B is a consequence of the usual duality theorems for the mixed norm spaces $L^{p}\left(l^{q}\right)$, and the Hahn-Banach theorem. We do not know whether there is a similar proof of Theorem A, or a dual version of part (ii) of Theorem A. (It follows from some recent results of N.J. Kalton and S.J. Montgomery-Smith [12] that the sufficient condition $\inf _{i \in I}\left\|F^{-1 / p} \phi_{i}\right\|_{L^{r, 1}\left(d \omega_{0}\right)}>0$ is not necessary for (1.5) in the case $1 \leq q<r$.)

We observe that the characterizations given in Theorems A and B are implicit. In the following theorem we give some sufficient conditions in order that (1.4) or (1.5) hold for an arbitrary family of functions $\left\{\phi_{i}\right\}$, and a wide range of $p, q$ and $r$.

Let $0<p<\infty, 0<r \leq \infty$ and $0<q \leq \infty$. Without loss of generality we may assume $\phi_{i} \in L^{p}(d \omega), \phi_{i} \geq 0$. We set

$F_{0}(x)= \begin{cases}{\left[\sup _{i}\left(\phi_{i}^{r-p}(x)\left\|\phi_{i}\right\|_{L^{p}(d \omega)}^{p}\right)\right]^{p /(r-p)},} & \text { if } 0<r \leq q \leq \infty, \\ {\left[\sum_{i}\left(\phi_{i}^{r-p}(x)\left\|\phi_{i}\right\|_{L^{p}(d \omega)}^{p}\right)^{q /(r-q)}\right]^{(r-q) p / q(r-p)},} & \text { if } 0<q<r<\infty,\end{cases}$

in the case $p<r$, and

$F_{0}(x)= \begin{cases}{\left[\sup _{i}\left(\phi_{i}^{p-r}(x) /\left\|\phi_{i}\right\|_{L^{p}(d \omega)}^{p}\right)\right]^{p /(p-r)},} & \text { if } 0<q \leq r<\infty, \\ {\left[\sum_{i}\left(\phi_{i}^{p-r}(x) /\left\|\phi_{i}\right\|_{L^{p}(d \omega)}^{p}\right)^{q /(q-r)}\right]^{(q-r) p / q(p-r)},} & \text { if } 0<r<q \leq \infty,\end{cases}$

in the case $p>r$.

Note that if $r \leq q$ in (1.12), or $q \leq r$ in (1.13), then $F_{0}(x)$ is a generalized maximal function associated with the family $\left\{\phi_{i}(x)\right\}$.

\section{Theorem 1.}

(i) Let $0<p<r<\infty$, and let $F_{0}$ be defined by (1.12). Then (1.4) holds if $F_{0} \in L^{1}(d \omega)$.

(ii) Let $0<r<p<\infty$, and let $F_{0}$ be defined by (1.13). Then (1.5) holds if $F_{0} \in L^{1}(d \omega)$. 
Theorem 1, along with some other vector-valued inequalities of this type, is proved in Sec. 2. In Sec. 3 we show that for functions $\left\{\phi_{i}\right\}$ that are constant multiples of characteristic functions of dyadic cubes the converse to Theorem 1 is also true.

Theorem 2. Let $\left\{Q_{i}\right\}$ be a family of dyadic cubes in $\mathbf{R}^{n}$. Suppose $\phi_{i}(x)=$ $c_{i} \chi_{Q_{i}}(x)$, where $\left\{c_{i}\right\}$ is a fixed sequence of non-negative numbers.

(i) Let $0<p<r<\infty$. Then (1.4) holds if and only if $F_{0} \in L^{1}(d \omega)$, where $F_{0}$ is defined by (1.12).

(ii) Let $0<r<p<\infty$. Then (1.5) holds if and only if $F_{0} \in L^{1}(d \omega)$, where $F_{0}$ is defined by (1.13).

Some special cases of Theorem 2 (i) were obtained in [21]. Similar "upper triangle" inequalities of type (1.4) in the case $q<p<r$ and $d \omega=d x$ are due to E. Amar and A. Bonami [2]. See also [14] and [15] where some inequalities of type (1.5) are given for $d \omega=d x$ with different proofs.

Theorem 2 yields a characterization of both forward and reverse imbeddings of $\mathbf{f}_{p}^{\alpha q}(\omega)$ spaces into $l^{r}$ spaces with arbitrary weights. We observe that Theorems 1 and 2, as well as Theorems A and B stated above, cover the so-called "upper triangle" case, where $p<r$ in (1.4), or $p>r$ in (1.5). In this case the "only if" statements are usually more difficult to prove than the "if" counterparts.

In the "classical" case, where $p \geq r$ in (1.4), or $p \leq r$ in (1.5), the corresponding results are equivalent via duality to dyadic versions of the Carleson measure theorem. (See Theorems 3 and 4 below.)

In Sec. 4 we consider a more difficult problem of characterizing multipliers for a pair of $\mathbf{f}$ spaces. We show that this is equivalent to certain two weight inequalities for general dyadic maximal or integral operators. (See [19]-[22].)

We wish to thank Professors William Cohn, Michael Frazier and Nigel Kalton for valuable discussions.

\section{Some remarks on vector-valued inequalities.}

In this section, we discuss some dual versions of the inequalities (1.4) and (1.5), and give a proof of Theorem 1 and Theorem B.

Let $J$ be any countable (or finite) subset of the index set $I$. For a positive locally finite measure $\omega$, we denote by $L^{p}\left(l^{q}, d \omega\right.$ ) (sometimes we omit $d \omega$ in this notation) the space of vector-valued functions $g=\left\{g_{j}\right\}_{j \in J}$ such that

$$
\|g\|_{L^{p}\left(l^{q}\right)}=\left[\int\left(\sum_{j}\left|g_{j}\right|^{q}\right)^{p / q} d \omega\right]^{1 / p}<\infty
$$


with the usual convention

$$
\|g\|_{L^{p}\left(l^{\infty}\right)}=\left[\int\left(\sup _{j}\left|g_{j}\right|\right)^{p} d \omega\right]^{1 / p}
$$

in case $q=\infty$. Suppose $0<p<\infty, 0<r<\infty$, and $0<q \leq \infty$. For any fixed sequence of real-valued functions $\left\{\phi_{j}\right\}_{j \in J}$ and $t>0$, we set

$$
\tilde{\phi}_{j}(x)=\phi_{j}^{t}(x), \quad \tilde{p}=p / t, \quad \tilde{q}=q / t, \quad \tilde{r}=r / t .
$$

Lemma 1. Let $0<p<\infty, 0<r \leq \infty$, and $0<q \leq \infty$. Then for any $t(0<t<\infty)$ such that $t \leq \min (q, r)$, and $t<p$, inequality (1.4) holds if and only if the inequality

$$
\left(\sum_{j}\left|\int \tilde{\phi}_{j} g_{j} d \omega\right|^{\tilde{r}^{\prime}}\right)^{1 / \tilde{r}^{\prime}} \leq C\|g\|_{L^{\tilde{p}^{\prime}}\left(l \tilde{q}^{\prime}\right)}
$$

holds for all $g=\left\{g_{j}\right\}_{j \in J}, J \subset I$, with $C$ independent of $g$ and $J$. (Here $1 / s+1 / s^{\prime}=1,1 \leq s \leq \infty$.)

Proof. Note that (1.4) is "invariant" under the transformation (2.1). In other words, it remains true (with a constant $C^{t}$ in place of $C$ ) if one replaces $\phi$ and $p, q, r$ by, respectively, $\tilde{\phi}$ and $\tilde{p}, \tilde{q}, \tilde{r}$, for any $t>0$. Since $0<t \leq \min (p, q, r)$, we have $\tilde{p} \geq 1, \tilde{q} \geq 1, \tilde{r} \geq 1$. By duality for spaces with mixed norms, $L^{\tilde{p}}\left(l^{\tilde{q}}, \omega\right)^{*}=L^{\tilde{p}^{\prime}}\left(l^{\tilde{q}^{\prime}}, \omega\right)$. Hence (1.4) is equivalent to the inequality

$$
\left|\int \sum_{j} s_{j} \tilde{\phi}_{j}(x) g_{j}(x) d \omega\right| \leq C\|s\|_{l^{\tilde{r}}}\|g\|_{L^{\tilde{p}^{\prime}}\left(l \tilde{q}^{\prime}\right)} .
$$

Using duality again, we see that this is equivalent to (2.2). The proof of Lemma 1 is complete.

Remark 1. In the case $0<q \leq \min (p, r)$, there is another dual form of (1.4). Letting $t=q$, so that $\tilde{q}^{\prime}=\infty$, and $g=\sup _{j}\left|g_{j}\right|$, we see that (2.2) holds if and only if

$$
\left(\sum_{j}\left|\int \phi_{j}^{q} g d \omega\right|^{\tilde{r}^{\prime}}\right)^{1 / \tilde{r}^{\prime}} \leq C\|g\|_{L_{\tilde{p}^{\prime}}(d \omega)},
$$

for all functions $g \in L^{\tilde{p}^{\prime}}(d \omega)$, where $\tilde{p}^{\prime}=p /(p-q)$ and $\tilde{r}^{\prime}=r /(r-q)$.

The following statement shows that reverse estimates of the form (1.5) can be derived from the forward ones. 
Corollary 1. Let $0<p<\infty, 0<r \leq \infty, 0<q \leq \infty$, and $0<t \leq$ $\min (q, r), t<p$. Let $\psi_{i}(x)=\phi_{i}^{p-t}(x) /\left\|\phi_{i}\right\|_{L^{p}(d \omega)}^{p}$. Then (1.5) holds if

$$
\left\|\left(\sum_{j}\left|s_{j}\right|^{\tilde{q}^{\prime}} \psi_{j}^{\tilde{q}^{\prime}}\right)^{1 / \tilde{q}^{\prime}}\right\|_{L^{\tilde{p}^{\prime}}(d \omega)} \leq C\|s\|_{l^{\tilde{r}^{\prime}}}
$$

for any finitely supported $s=\left\{s_{j}\right\}$. Here $\tilde{p}, \tilde{q}, \tilde{r}$ are defined by (2.1).

Proof. By Lemma 1, it follows that (2.4) is equivalent to the estimate

$$
\left(\sum_{j}\left|\int \psi_{j} g_{j} d \omega\right|^{\tilde{r}}\right)^{1 / \tilde{r}} \leq C\|g\|_{L^{\tilde{p}}\left(l^{\tilde{q}}\right)},
$$

for all $g \in L^{\tilde{p}}\left(l^{\tilde{q}}, \omega\right)$. Letting $g=\left\{s_{j} \phi_{j}^{t}\right\}$ in (2.5), and taking into account that $\int \psi_{j} g_{j} d \omega=s_{j}$, we get (1.5). The proof of Corollary 1 is complete.

In the following lemma we list the cases where an explicit characterization of (1.4) and (1.5) is possible.

\section{Lemma 2.}

(a) If $0<r \leq \min (p, q)$, then (1.4) holds if and only if $\sup _{i}\left\|\phi_{i}\right\|_{L^{p}(d \omega)}<$ $\infty$.

(b) If $\max (p, q) \leq r \leq \infty$, then (1.5) holds if and only if $\inf _{i}\left\|\phi_{i}\right\|_{L^{p}(d \omega)}>$ 0.

(c) If $q=p<r$, then (1.4) holds if and only if $\sum_{i}\left\|\phi_{i}\right\|_{L^{p}(d \omega)}^{r p /(r-p)}<\infty$.

(d) If $q=p>r$, then (1.5) holds if and only if $\sum_{i}\left\|\phi_{i}\right\|_{L^{p}(d \omega)}^{-r p /(p-r)}<\infty$.

(e) If $r=\infty$, then (1.4) holds if and only if $\left\|\left(\sum_{i} \phi_{i}^{q}\right)^{1 / q}\right\|_{L^{p}(d \omega)}<\infty$.

For other values of $p, q, r$, a complete characterization of (1.4) or (1.5) seems to be nontrivial.

Proof of Lemma 2. (a) Suppose $\sup _{i}\left\|\phi_{i}\right\|_{L^{p}(d \omega)}<\infty$. If $0<q \leq p$, then by the integral Minkowski's inequality

$$
\begin{aligned}
\left\|\left(\sum_{i}\left|s_{i}\right|^{q} \phi_{i}^{q}\right)^{1 / q}\right\|_{L^{p}(d \omega)} & \leq\left(\sum_{i}\left(\int\left|s_{i}\right|^{p} \phi_{i}^{p} d \omega\right)^{q / p}\right)^{1 / q} \\
& =\left(\sum_{i}\left|s_{i}\right|^{q}\left\|\phi_{i}\right\|_{L^{p}(d \omega)}^{q}\right)^{1 / q} \leq C\|s\|_{l^{r}} .
\end{aligned}
$$


The last inequality holds since $q \geq r$. In the case $p<q \leq \infty$, we have

$$
\begin{aligned}
\left\|\left(\sum_{i}\left|s_{i}\right|^{q} \phi_{i}^{q}\right)^{1 / q}\right\|_{L^{p}(d \omega)} & \leq\left\|\left(\sum_{i}\left|s_{i}\right|^{p} \phi_{i}^{p}\right)^{1 / p}\right\|_{L^{p}(d \omega)} \\
& =\left(\sum_{i}\left|s_{i}\right|^{p}\left\|\phi_{i}\right\|_{L^{p}(d \omega)}^{p}\right)^{1 / p} \leq C\|s\|_{l^{r}},
\end{aligned}
$$

since $p \leq r$. Thus (1.4) holds. The converse statement is obvious.

Statement (b) can be proved in a similar manner, or derived from (a). Indeed, suppose $\inf _{i}\left\|\phi_{i}\right\|_{L^{p}(d \omega)}>0$. Choose $0<t<\min (p, q, r)$ and set $\tilde{p}=p / t$. Define $\psi_{\imath}(x)=\phi_{i}^{p-t}(x) /\left\|\phi_{i}\right\|_{L^{p}(d \omega)}^{p}$, as in the proof of Corollary 1 . Then $\sup _{i}\left\|\psi_{i}\right\|_{L^{p /(p-t)}(d \omega)}=\sup _{i}\left\|\phi_{i}\right\|_{L^{p}(d \omega)}^{-t}<\infty$. By part (a) we conclude that (2.4) holds, which implies (1.5) by Corollary 1 . The converse statement is obvious, as well as the remaining cases (c), (d), and (e). The proof of Lemma 2 is complete.

Now we prove Theorem 1(i). Suppose $0<p<r<\infty$. From Lemma 1 it follows that we may assume $p>1, q>1, r>1$. Suppose $F_{0} \in L^{1}(d \omega)$, where $F_{0}$ is given by (1.12). Let $g=\left\{g_{j}\right\} \in L^{p^{\prime}}\left(l^{q^{\prime}}, d \omega\right)$. By Hölder's inequality with exponents $r$ and $r^{\prime}$, we have

$$
\left|\int \phi_{j} g_{j} d \omega\right|^{r^{\prime}} \leq\left(\int\left|\phi_{j}\right|^{p} d \omega\right)^{r^{\prime}-1} \int\left|\phi_{j}\right|^{(r-p)\left(r^{\prime}-1\right)}\left|g_{j}\right|^{r^{\prime}} d \omega .
$$

Hence

$$
\sum_{j}\left|\int \phi_{j} g_{j} d \omega\right|^{r^{\prime}} \leq \int \sum\left|g_{j}\right|^{r^{\prime}} \psi_{j} d \omega
$$

where

$$
\psi_{j}(x)=\phi_{j}^{(r-p)\left(r^{\prime}-1\right)}(x)\left\|\phi_{j}\right\|_{L^{p}(d \omega)}^{p\left(r^{\prime}-1\right)} .
$$

We estimate the right hand side of the preceding inequality. Let $F_{0}$ be defined by (1.12). If $q \geq r$, then $F_{0}(x)=\sup _{j} \psi_{j}^{p(r-1) /(r-p)}(x)$. By Hölder's inequality with exponents $p^{\prime} / r^{\prime}$ and $\left(p^{\prime} / r^{\prime}\right)^{\prime}=p(r-1) /(r-p)$ we get

$$
\begin{aligned}
\int \sum_{j}\left|g_{j}\right|^{r^{\prime}} \psi_{j} d \omega & \leq \int \sup _{j} \psi_{j} \sum_{j}\left|g_{j}\right|^{r^{\prime}} d \omega \\
& \leq\|g\|_{L^{p^{\prime}\left(l^{r^{\prime}}\right)}}^{r^{\prime}}\left(\int F_{0} d \omega\right)^{(r-p) / p(r-1)}
\end{aligned}
$$




$$
\leq\|q\|_{L^{p^{\prime}}\left(l^{q^{\prime}}\right)}^{r^{\prime}}\left(\int F_{0} d \omega\right)^{(r-p) / p(r-1)}
$$

The last inequality holds since $q^{\prime} \leq r^{\prime}$.

Analogously, if $q<r$, then $q^{\prime}>r^{\prime}$. Applying Hölder's inequality twice, we obtain

$$
\begin{aligned}
\int \sum_{j}\left|g_{j}\right|^{r^{\prime}} \psi_{j} d \omega & \leq \int\left(\sum_{j}\left|g_{j}\right|^{q^{\prime}}\right)^{r^{\prime} / q^{\prime}}\left(\sum_{j} \psi_{j}^{q(r-1) /(r-q)}\right)^{1-r^{\prime} / q^{\prime}} d \omega \\
& \leq\|g\|_{L^{p^{\prime}}\left(l^{q^{\prime}}\right)}^{r^{\prime}}\left(\int F_{0} d \omega\right)^{(r-p) / p(r-1)} .
\end{aligned}
$$

Thus we have

$$
\sum_{j}\left|\int \phi_{j} g_{j} d \omega\right|^{r^{\prime}} \leq C\|g\|_{L^{p^{\prime}\left(q^{\prime}\right)}}^{r^{\prime}} .
$$

By Lemma 1, this completes the proof of Theorem 1 (i).

Now we prove statement (ii) of Theorem 1 . Suppose $p>r$, and $F_{0} \in$ $L^{1}(d \omega)$, where $F_{0}$ is defined by (1.13). Since (1.5) is "invariant" under the transform (2.1), we can assume $p, q, r>1$, as in the proof of (i). (Otherwise, we choose $0<t<\min (p, q, r)$, and replace $\phi_{i}$ and $p, q, r$ by, respectively, $\tilde{\phi}_{i}$ and $\tilde{p}, \tilde{q}, \tilde{r}$.

We set $\psi_{j}(x)=\phi_{j}^{p-1}(x) /\left\|\phi_{j}\right\|_{L^{p}(d \omega)}^{p}$. It is easily seen that

$$
\frac{\phi_{j}^{p}(x)}{\left(\left\|\phi_{j}\right\|_{L^{p}(d \omega)}^{p}\right)^{p /(p-r)}} \equiv \frac{\psi_{j}^{p^{\prime}}(x)}{\left(\left\|\psi_{j}\right\|_{L^{p^{\prime}}(d \omega)}^{p^{\prime}}\right)^{p^{\prime} /\left(p^{\prime}-r^{\prime}\right)}} .
$$

(Note that $p^{\prime}<r^{\prime}$.) From the preceding inequality it follows that the function $F_{0}(x)$ defined by $(1.13)$ can be rewritten as

$$
F_{0}(x)= \begin{cases}{\left[\sup _{j}\left(\psi_{j}^{r^{\prime}-p^{\prime}}(x)\left\|\psi_{j}\right\|_{L^{p^{\prime}}(d \omega)}^{p^{\prime}}\right)\right]^{p^{\prime} /\left(r^{\prime}-p^{\prime}\right)},} & \text { if } 0<q \leq r \\ {\left[\sum_{j}\left(\psi_{j}^{r^{\prime}-p^{\prime}}(x)\left\|\psi_{j}\right\|_{L^{p^{\prime}(d \omega)}}^{p^{\prime}}\right)^{q^{\prime} /\left(r^{\prime}-q^{\prime}\right)}\right]^{\left(r^{\prime}-q^{\prime}\right) p^{\prime} / q^{\prime}\left(r^{\prime}-p^{\prime}\right)},} & \text { if } 0<r<q .\end{cases}
$$

Since $F_{0} \in L^{1}(d \omega)$, then by part (i) of Theorem 1 proved above we have

$$
\left\|\left(\sum_{j}\left|s_{j}\right|^{q^{\prime}} \psi_{j}^{q^{\prime}}\right)^{1 / q^{\prime}}\right\|_{L^{p^{\prime}}(d \omega)} \leq C\|s\|_{l^{r^{\prime}}}
$$


for all $s=\left\{s_{j}\right\}$. By Corollary 1 (with $t=1$ ), this implies (1.5). The proof of Theorem 1 is complete.

The proof of the following lemma is based on the Hahn-Banach Theorem.

Lemma 3. Suppose $0<r<p<\infty$ and $0<r \leq q \leq \infty$. Let $p_{1}=p /(p-r)$ and $q_{1}=q /(q-r)$. Then (1.5) holds if and only if there exists $g=\left\{g_{i}\right\}$ such that $\|g\|_{L^{p_{1}\left(l^{q_{1}}\right)}} \leq 1$, and

$$
\inf _{i} \int\left|g_{i}\right| \phi_{i}^{r} d \omega>0
$$

Remark 2. If $0<q=r<p<\infty$, then setting $g(x)=\sup _{i}\left|g_{i}(x)\right|$ and

$$
F(x)=\left(|g(x)| /\|g\|_{L^{p /(p-r)}(d \omega)}\right)^{p /(p-r)},
$$

we see from (2.6) that there exists $F$ such that

$$
\int F d \omega \leq 1, \quad \text { and } \quad \inf _{i}\left\|F^{-1 / p} \phi_{i}\right\|_{L^{r}(F d \omega)}>0 .
$$

This proves the "only if" part of Theorem B (see the Introduction; the "if" part of Theorem B is obvious).

Proof of Lemma 3. Suppose $0<r<p$. As in the proof of Theorem 1, we make use of the fact that (1.5) is "invariant" under the transform (2.1). Letting $t=r$ in (2.1), and replacing $p, q$ and $\phi_{i}$ by, respectively, $\tilde{p}=p / r, \tilde{q}=$ $q / r$, and $\psi_{i}=\phi_{i}^{r}$, we may rewrite $(1.5)$ as

$$
\left\|\left(\sum_{i \in I}\left|s_{i}\right|^{\tilde{q}} \psi_{i}^{\tilde{q}}\right)^{1 / \tilde{q}}\right\|_{L^{\tilde{p}}(d \omega)} \geq C\left|\sum_{i} s_{i}\right| .
$$

Let $\mathcal{L}$ denote the subspace of $L^{\tilde{p}}\left(l^{\tilde{q}}, \omega\right)$ consisting of all finitely supported vector functions of the form $s=\left\{s_{i} \psi_{i}\right\}_{i \in I}$, for all real $\left\{s_{i}\right\}$. The preceding inequality means that the linear functional defined on $\mathcal{L}$ by $G\left\{s_{i} \psi_{i}\right\}=\sum_{i} s_{i}$ is bounded. Let $\mathcal{B}=\left[L^{\tilde{p}}\left(l^{\tilde{q}}, \omega\right)\right]^{*}$. Since $\tilde{p}>1$, we have $\mathcal{B}=L^{p_{1}}\left(l^{q_{1}}, \omega\right)$ if $q>r$, and $\mathcal{B}=L^{p_{1}}\left(l^{\infty}, \omega\right)$ if $q=r$. By the Hahn-Banach theorem, there is an extension $\tilde{G}=\left\{g_{i}\right\}_{i \in I}$ of $G$ defined on $\mathcal{B}$ so that $\|\tilde{G}\|_{\mathcal{B}} \leq 1 / C$, where $C$.is the constant in (1.5), and $\tilde{G}$ coincides with $G$ on $\mathcal{L}$. The latter means that

$$
\int \sum_{i} s_{i} g_{i} \psi_{i} d \omega=\sum_{i} s_{i}
$$


for all finitely supported $s_{i}$, i.e., $\int g_{i} \psi_{i} d \omega=1$ for all $i \in I$. Hence

$$
\inf _{i} \int\left|g_{i}\right| \psi_{i} d \omega=\inf _{i} \int\left|g_{i}\right| \phi_{i}^{r} d \omega \geq 1
$$

Setting $g=C \tilde{G}$, we see that $\|g\|_{\mathcal{B}} \leq 1$, and (2.6) holds.

Conversely, suppose there is a $g=\left\{g_{i}\right\}$ such that $\|g\|_{L^{p_{1}\left(l^{q_{1}}\right)}} \leq 1$, where $p_{1}=p /(r-p), q_{1}=q /(q-r)$, and (2.6) holds. Then using (2.6) and applying Hölder's inequality twice we get

$$
\sum_{i}\left|s_{i}\right|^{r} \leq C \int \sum_{i}\left|s_{i}\right|^{r} \phi_{i}^{r}\left|g_{i}\right| d \omega \leq C\left\|\left(\sum_{i}\left|s_{i}\right|^{q} \psi_{i}^{q}\right)^{1 / q}\right\|_{L^{p}(d \omega)}^{r} .
$$

The proof of Lemma 3 is complete.

Remark 3. Lemma 3 remains true for $r=p<q \leq \infty$ (the case $r=p \geq q$ follows from Lemma 2). If $r=p$ and $q=\infty$, there is a deeper version of Lemma 3 due to L. Dor [4]. We can set $g=\left\{\chi_{E_{i}}\right\}$ in (2.6), where $\left\{E_{i}\right\}_{i \in I}$ $\left(E_{i} \subset X\right)$ is a family of disjoint sets. Thus, for all $0<r<\infty$, the inequality

$$
\int_{X} \sup _{i \in I}\left(\left|s_{i}\right|^{r} \phi_{i}^{r}\right) d \omega \geq C \sum_{i}\left|s_{i}\right|^{r}
$$

holds if and only if there exists a family of disjoint sets $\left\{E_{i}\right\}$ so that

$$
\inf _{i} \int_{E_{i}} \phi_{i}^{r} d \omega \geq C
$$

This result for the functions $\left\{\phi_{Q}\right\}_{Q \in \mathcal{Q}}$ has interesting connections with the dyadic Carleson measure theorem discussed in the next section (see [17], and also Corollary 2 below).

\section{Imbedding theorems for $f$ spaces.}

Let $\mathcal{Q}=\{Q\}$ be the family of all dyadic cubes in $\mathbf{R}^{n}$. To any $Q \in \mathcal{Q}$ we associate a fixed non-negative number $c_{Q}$. Let $\phi_{Q}(x)=c_{Q} \chi_{Q}(x)$, for all $x \in \mathbf{R}^{n}$. Note that

$$
\sum_{Q} \phi_{Q}(x)=\sum_{x \in Q} c_{Q}
$$

where the sum on the right-hand side is taken over all $Q$ containing $x$.

The following theorem generalizes Theorem 2 (i) and gives a characterization of the imbeddings of weighted $l^{r}$ spaces into $\mathbf{f}_{p}^{\alpha q}(\omega)$ spaces. (See the Introduction.) 
Theorem 3. Let $0<p<\infty, 0<r<\infty$, and $0<q \leq \infty$. Let $\omega$ be a positive locally finite measure on $\mathbf{R}^{n}$. The inequality

$$
\left\|\left(\sum_{x \in Q}\left|s_{Q}\right|^{q} c_{Q}^{q}\right)^{1 / q}\right\|_{L^{p}(d \omega)} \leq C\|s\|_{l^{r}}
$$

holds for all scalar sequences $s=\left\{s_{Q}\right\}$ if and only if one of the following conditions holds.

(a) $0<r \leq \min (p, q)$, and

$$
\sup _{Q} c_{Q}|Q|_{\omega}^{1 / p}<\infty
$$

(b) $0<q<r \leq p$, and for all dyadic cubes $P$,

$$
\sum_{Q \subset P}\left(c_{Q}^{q}|Q|_{\omega}\right)^{r /(r-q)} \leq C|P|_{\omega}^{r(p-q) / p(r-q)}
$$

(c) $\max (p, q)<r<\infty$, and

$$
\int\left[\sum_{x \in Q}\left(c_{Q}^{r}|Q|_{\omega}\right)^{q /(r-q)}\right]^{p(r-q) / q(r-p)} d \omega<\infty .
$$

(d) $0<p<r \leq q \leq \infty$, and

$$
\int \sup _{x \in Q}\left(c_{Q}^{r}|Q|_{\omega}\right)^{p /(r-p)} d \omega<\infty
$$

Proof. Statement (a) follows from Lemma 2. In case (b), we use a dual characterization given by (2.3). For the family $\left\{\phi_{Q}\right\}$, where $\phi_{Q}=c_{Q} \chi_{Q}$, the latter boils down to the inequality

$$
\left(\sum_{Q}\left(c_{Q}^{q}|Q|_{\omega}\right)^{r /(r-q)}\left|\frac{1}{|Q|_{\omega}} \int_{Q} g d \omega\right|^{r /(r-q)}\right)^{(r-q) / r} \leq C\|g\|_{L^{p /(p-q)}(d \omega)} .
$$

We observe that (3.6) is a dyadic form of the Carleson inequality (see [17], [19]); and (3.3) is a discrete version of the Carleson-Duren condition (see [10]). The necessity of (3.3) for (3.6) is obvious (use a test function $g=\chi_{P}$ ); sufficiency is proved, for any measure $\omega$, using a standard technique involving weak type estimates and interpolation (see [9], [19]). 
Now we prove statement (c). It follows from Theorem 1 (i) that (3.4) $\Rightarrow$ (3.1). Indeed, for the family $\phi_{Q}=c_{Q} \chi_{Q}$, the function $F_{0}$ defined by (1.12) is in $L^{1}(d \omega)$ if and only if (3.4) holds.

To prove $(3.1) \Rightarrow(3.4)$, we will need an analogue of the Fefferman-Stein vector-valued maximal inequality [5] for the dyadic maximal operator

$$
M_{\omega}^{d} f(x)=\sup _{x \in Q} \frac{1}{|Q|_{\omega}} \int_{Q}|f| d \omega
$$

where $\omega$ is an arbitrary locally finite measure on $\mathbf{R}^{n}$. (The supremum in (3.7) is taken over all $Q \in \mathcal{Q}_{0}$ containing $x$.)

Theorem C. Let $1<p<\infty$ and $1<q \leq \infty$. Suppose $\omega$ is a locally finite measure on $\mathbf{R}^{n}$. Then

$$
\left\|\left(\sum_{j}\left(M_{\omega}^{d} f_{j}\right)^{q}\right)^{1 / q}\right\|_{L^{p}(d \omega)} \leq C\left\|\left(\sum_{j}\left|f_{j}\right|^{q}\right)^{1 / q}\right\|_{L^{p}(d \omega)},
$$

for all $\left\{f_{j}\right\} \in L^{p}\left(l^{q}, d \omega\right)$.

Theorem $\mathrm{C}$ is possibly known, but we were not able to find a reference and sketch a proof below. (As was pointed out by the referee, the original proof given in [5] in the case $d \omega=d x$ should be modified, at least in the case $p<q$, if $\omega$ does not have a doubling property.) We note that in the case $p \geq q$ the proof of Theorem C given in [5] for $d \omega=d x$ basically remains valid for $M_{\omega}^{d}$ with arbitrary $\omega$. The idea is to use duality together with a weighted inequality for the scalar maximal function (Lemma $\mathrm{D}$ below). Then we reduce the case $p<q$ to $p \geq q$ by means of a more sophisticated duality argument, making use of Lemma D again. (In [5], for $p<q$ and $d \omega=d x,(3.8)$ is derived from a weak type vector-valued estimate based on the Calderón-Zygmund lemma.)

Proof. We will need the following weighted inequality.

Lemma D. Let $1<r \leq \infty$. Let $\omega$ be a locally finite measure on $\mathbf{R}^{n}$. Then

$$
\int\left(M_{\omega}^{d} f\right)^{r} \phi d \omega \leq C \int|f|^{r}\left(M_{\omega}^{d} \phi\right) d \omega
$$

where $C$ does not depend on $f$ and $\phi\left(\phi \geq 0, \phi \in L_{\text {loc }}^{1}(d \omega)\right)$.

The proof of Lemma D is similar to that given in [5] in case $d \omega=d x$ and is outlined below for the sake of convenience. 
Proof. We prove the following weak type estimate. Let $t>0$ and $E_{t}=\{x$ : $\left.M_{\omega}^{d} f(x) \geq t\right\}$. Then

$$
\int_{E_{t}} \phi d \omega \leq \frac{1}{t} \int|f| M_{\omega}^{d} \phi d \omega .
$$

First we assume that all dyadic cubes have side length less than $N$. Then $E_{t}=\cup Q$, where $Q$ are pairwise disjoint (maximal) dyadic cubes such that $|Q|_{\omega}^{-1} \int_{Q}|f| d \omega \geq t$. (See [19].) As in [5], for all $Q$ in this collection we have

$$
t \int_{Q} \phi d \omega \leq\left(|Q|_{\omega}^{-1} \int_{Q}|f| d \omega\right) \int_{Q} \phi d \omega \leq \int_{Q}|f| M_{\omega}^{d} \phi d \omega
$$

Thus

$$
t \int_{E_{t}} \phi d \omega=\sum_{Q} t \int_{Q} \phi d \omega \leq \int|f| M_{\omega}^{d} \phi d \omega
$$

Letting $N \rightarrow \infty$, we obtain the weak type estimate. Then (3.9) follows by a standard interpolation argument for all $1<r<\infty$, since it is obviously true for $r=\infty$. The proof of Lemma D is complete.

If $p \geq q$, then we complete the proof of Theorem $\mathrm{C}$ by applying Lemma $\mathrm{D}$ with $r=p /(p-q)$, as in [5]:

$$
\begin{aligned}
& \left\|\left(\sum_{j}\left(M_{\omega}^{d} f_{j}\right)^{q}\right)^{1 / q}\right\|_{L^{p}(d \omega)}^{q} \\
& =\sup \left\{\int \sum_{j}\left(M_{\omega}^{d} f_{j}\right)^{q} \phi d \omega:\|\phi\|_{L^{p /(p-q)}(d \omega)} \leq 1\right\} \\
& \leq C \sup \left\{\int \sum_{j}\left|f_{j}\right|^{q} M_{\omega}^{d} \phi d \omega:\|\phi\|_{L^{p /(p-q)}(d \omega)} \leq 1\right\} .
\end{aligned}
$$

Since $M_{\omega}^{d}$ in bounded in $L^{p /(p-q)}(d \omega)$, we have

$$
\left\|M_{\omega}^{d} \phi\right\|_{L^{p /(p-q)}(d \omega)} \leq C\|\phi\|_{L^{p /(p-q)}(d \omega)} \leq C .
$$

Then by Hölder's inequality with exponents $p / q$ and $p /(p-q)$ we get

$$
\int \sum_{j}\left|f_{j}\right|^{q} M_{\omega}^{d} \phi d \omega \leq C\left\|\left(\sum_{j}\left|f_{j}\right|^{q}\right)^{1 / q}\right\|_{L^{p}(d \omega)}^{q} .
$$


Thus

$$
\left\|\left(\sum_{j}\left(M_{\omega}^{d} f_{j}\right)^{q}\right)^{1 / q}\right\|_{L^{p}(d \omega)} \leq C\left\|\left(\sum_{j}\left|f_{j}\right|^{q}\right)^{1 / q}\right\|_{L^{p}(d \omega)}
$$

which proves Theorem $\mathrm{C}$ in the case $p \geq q$.

Now suppose $p<q$. We choose $1<r<p$. Then by the duality relation

$$
\left[L^{p / r}\left(l^{q / r}, \omega\right)\right]^{*}=L^{p /(p-r)}\left(l^{q /(q-r)}, \omega\right)
$$

we have

$$
\begin{aligned}
& \left\|\left(\sum_{j}\left(M_{\omega}^{d} f_{j}\right)^{q}\right)^{1 / q}\right\|_{L^{p}(d \omega)}^{r} \\
& =\sup \left\{\int \sum_{j}\left(M_{\omega}^{d} f_{j}\right)^{r}\left|\phi_{j}\right| d \omega:\left\|\left\{\phi_{j}\right\}\right\|_{\left.L^{p /(p-r)\left(l^{q /(q-r)}\right)} \leq 1\right\} .}\right.
\end{aligned}
$$

Applying Lemma D again, we get

$$
\int \sum_{j}\left(M_{\omega}^{d} f_{j}\right)^{r}\left|\phi_{j}\right| d \omega \leq C \int \sum_{j}\left|f_{j}\right|^{r} M_{\omega}^{d} \phi_{j} d \omega .
$$

By Hölder's inequality,

$$
\int \sum_{j}\left|f_{j}\right|^{r} M_{\omega}^{d} \phi_{j} d \omega \leq\left\|\left\{f_{j}\right\}\right\|_{L^{p}\left(l^{q}\right)}^{r}\left\|\left\{M_{\omega}^{d} \phi_{j}\right\}\right\|_{L^{p /(p-r)}\left(l^{q /(q-r)}\right)}
$$

Since $p /(p-r)>q /(q-r)$, it follows from the case considered above

$$
\left\|\left\{M_{\omega}^{d} \phi_{j}\right\}\right\|_{L^{p /(p-r)(l q /(q-r))}} \leq C\left\|\left\{\phi_{j}\right\}\right\|_{L^{p /(p-r)\left(l^{q /(q-r)}\right)}} \leq C .
$$

Thus

$$
\left\|\left(\sum_{j}\left(M_{\omega}^{d} f_{j}\right)^{q}\right)^{1 / q}\right\|_{L^{p}(d \omega)} \leq C\left\|\left(\sum_{j}\left|f_{j}\right|^{q}\right)^{1 / q}\right\|_{L^{p}(d \omega)}
$$

for $p<q$, which completes the proof of Theorem C. 
Now we return to the proof of Theorem 3 (c). We may assume again without loss of generality that $p>1, q>1$, and $r>1$. By Lemma 1, it follows that (3.1) is equivalent to the inequality

$$
\left(\sum_{Q} c_{Q}^{r^{\prime}}\left|\int_{Q} g_{Q} d \omega\right|^{r^{\prime}}\right)^{1 / r^{\prime}} \leq C\|g\|_{L^{p^{\prime}\left(l^{\prime}\right)}},
$$

for all $g=\left\{g_{Q}\right\} \in L^{p^{\prime}}\left(l^{q^{\prime}}\right)$. Since $r>\max (p, q)$, we have $r^{\prime}<\min \left(p^{\prime}, q^{\prime}\right)$. Setting $g_{Q}=\psi_{Q}^{1 / r^{\prime}}\left(\psi_{Q} \geq 0\right)$, and $\tilde{p}^{\prime}=p^{\prime} / r^{\prime}, \tilde{q}^{\prime}=q^{\prime} / r^{\prime}(\tilde{p}>1, \tilde{q}>1)$, we rewrite (3.10) in the equivalent form

$$
\sum_{Q}\left(c_{Q}|Q|_{\omega}\right)^{r^{\prime}}\left(|Q|_{\omega}^{-1} \int_{Q} \psi_{Q}^{1 / r^{\prime}} d \omega\right)^{r^{\prime}} \leq C\|\psi\|_{L^{\tilde{p}^{\prime}\left(\tilde{q}^{\prime}\right)}}
$$

for all $\psi=\left\{\psi_{Q}\right\} \in L^{\tilde{p}^{\prime}}\left(l^{\tilde{q}^{\prime}}, \omega\right)$.

By Hölder's inequality, obviously (3.11) holds if

$$
\sum_{Q}\left(c_{Q}|Q|_{\omega}\right)^{r^{\prime}}|Q|_{\omega}^{-1} \int_{Q} \psi_{Q} d \omega \leq C\|\psi\|_{L^{\tilde{p}^{\prime}}\left(l^{\tilde{q}^{\prime}}\right)}
$$

for all $\psi=\left\{\psi_{Q}\right\} \in L^{\tilde{p}^{\prime}}\left(l^{\tilde{q}^{\prime}}, \omega\right)$. Let us show that, conversely, (3.11) $\Rightarrow(3.12)$.

Let $\psi=\left\{\psi_{Q}\right\} \in L^{\tilde{p}^{\prime}}\left(l^{\tilde{q}^{\prime}}, \omega\right)$. Then, by Theorem C, $M_{\omega}^{d} \psi=\left\{M_{\omega}^{d} \psi_{Q}\right\} \in$ $L^{\tilde{p}^{\prime}}\left(l^{\tilde{q}^{\prime}}, \omega\right)$, and

$$
\left\|M_{\omega}^{d} \psi\right\|_{L^{\tilde{p}^{\prime}\left(\tilde{q}^{\prime}\right)}} \leq C\|\psi\|_{\left.L^{\tilde{p}^{\prime}\left(l \tilde{q}^{\prime}\right.}\right)} .
$$

Applying (3.11) with $M_{\omega}^{d} \psi$ in place of $\psi$, we obtain

$$
\begin{aligned}
& \sum_{Q}\left(c_{Q}|Q|_{\omega}\right)^{r^{\prime}}\left(|Q|_{\omega}^{-1} \int_{Q}\left(M_{\omega}^{d} \psi_{Q}\right)^{1 / r^{\prime}} d \omega\right)^{r^{\prime}} \\
& \leq C\left\|M_{\omega}^{d} \psi\right\|_{L^{\tilde{p}^{\prime}}\left(l \tilde{q}^{\prime}\right)} \leq C\|\psi\|_{L^{\tilde{p}^{\prime}}\left(\bar{l}^{\prime}\right)} .
\end{aligned}
$$

For all dyadic cubes $Q$ and $\psi_{Q} \geq 0$ clearly

$$
|Q|_{\omega}^{-1} \int_{Q} \psi_{Q} d \omega \leq\left(|Q|_{\omega}^{-1} \int_{Q}\left(M_{\omega}^{d} \psi_{Q}\right)^{1 / r^{\prime}} d \omega\right)^{r^{\prime}}
$$

Combining the preceding inequality and (3.13), we see that (3.12) holds. Thus (3.11) is equivalent to (3.12). It remains to note that by duality (3.12) can be rewritten in the form $\phi \in L^{\tilde{p}}\left(l^{\tilde{q}}, \omega\right)$, where $\phi=\left\{c_{Q}^{r^{\prime}}|Q|_{\omega}^{r^{\prime}-1} \chi_{Q}\right\}$, which coincides with (3.4). This completes the proof of statement (c) of Theorem 3 . 
In case (d) Theorem 3 was proved in [21]. For the sake of completeness, we give a sketch of the proof here. Note that, as in case (c), it follows that $(3.5) \Rightarrow(3.1)$ by Theorem 1 (i). To prove the converse, we make use of Pisier's lemma [Theorem A (ii) ]. Suppose (3.1) holds for $p<r$. Then there exists $F \in L^{1}(d \omega), F \geq 0$, such that

$$
\sup _{Q} c_{Q}\left\|F^{-1 / p} \chi_{Q}\right\|_{L^{r \infty}(F d \omega)}<\infty
$$

Set $f(x)=F^{-1 / p}(x) \chi_{Q}(x)$ and $d \nu=F d \omega$. Note that $\|f\|_{L^{p}(d \nu)}=|Q|_{\omega}^{1 / p}$. Suppose $p<s<r$, and $1 / s=t / p+(1-t) / r$, where $0<t<1$. Applying the elementary interpolation inequality

$$
\|f\|_{L^{s}(d \nu)} \leq C\|f\|_{L^{p}(d \nu)}^{t}\|f\|_{L^{r \infty}(d \nu)}^{1-t}
$$

we have

$$
\left(\int_{Q} F^{-s / p+1} d \omega\right)^{1 / s} \leq C|Q|_{\omega}^{t / p}\left\|F^{-1 / p} \chi_{Q}\right\|_{L^{r \infty}(F d \omega)}^{1-t}
$$

Letting $\beta=s / p-1>0$, and combining the preceding inequality with (3.14), we obtain

$$
\left(c_{Q}|Q|_{\omega}^{1 / r}\right)^{p r /(r-p)}\left(|Q|_{\omega}^{-1} \int_{Q} F^{-\beta} d \omega\right)^{1 / \beta} \leq C<\infty
$$

By Hölder's inequality,

$$
\left(|Q|_{\omega}^{-1} \int_{Q} F^{-\beta} d \omega\right)^{1 / \beta}\left(|Q|_{\omega}^{-1} \int_{Q} F^{\epsilon} d \omega\right)^{1 / \epsilon} \geq 1,
$$

for all $\epsilon>0$ and $\beta>0$. Hence

$$
\left(c_{Q}|Q|_{\omega}^{1 / r}\right)^{p r /(r-p)} \leq C\left(|Q|_{\omega}^{-1} \int_{Q} F^{\epsilon} d \omega\right)^{1 / \epsilon}
$$

and

$$
\int F_{0}(x) d \omega=\int \sup _{x \in Q}\left(c_{Q}|Q|_{\omega}^{1 / r}\right)^{p r /(r-p)} d \omega \leq C\left\|M_{\omega}^{d} F^{\epsilon}\right\|_{L^{1 / \epsilon}(d \omega)}^{1 / \epsilon}
$$

Suppose $0<\epsilon<1$. Since the dyadic maximal operator $M_{\omega}^{d}$ is bounded in $L^{1 / \epsilon}(d \omega)$, we get $F_{0} \in L^{1}(d \omega)$. The proof of Theorem 3 is complete. 
Remark 4. It follows from the proof of Theorem 3 that the best constant $C$ in (3.1) satisfies the inequality $A_{1} \kappa(c) \leq C \leq A_{2} \kappa(c)$, where $A_{1}, A_{2}$ are positive constants that do not depend on $c=\left\{c_{Q}\right\}$, and $\kappa(c)$ is defined in terms of the quantities given by (3.2)-(3.5): $\kappa(c)=\sup _{Q} c_{Q}|Q|_{\omega}^{1 / p}$ if $0<r \leq \min (p, q) ; \kappa(c)=C^{(r-q) / r q}$ if $q<r \leq p$, where $C$ is the best constant in (3.3);

$$
\kappa(c)=\left\|\left(\sum_{x \in Q}\left(c_{Q}|Q|_{\omega}^{1 / r}\right)^{q r /(r-q)}\right)^{(r-q) / q r}\right\|_{L^{p r /(r-p)}(d \omega)},
$$

if $\max (p, q)<r ;$ and

$$
\kappa(c)=\left\|\sup _{x \in Q}\left(c_{Q}|Q|_{\omega}^{1 / r}\right)\right\|_{L^{r p /(p-r)}(d \omega)}
$$

if $p<r \leq q$.

Now we state the following theorem which contains part (ii) of Theorem 2 , and yields a characterization of imbeddings of $f_{p}^{\alpha q}(\omega)$ spaces into weighted $l^{r}$ spaces.

Theorem 4. Let $0<p<\infty, 0<r<\infty$, and $0<q \leq \infty$. Suppose $\omega$ is a locally finite measure on $\mathbf{R}^{n}$, and $\left\{c_{Q}\right\}_{Q \in \mathcal{Q}}\left(c_{Q} \geq 0\right)$ is a fixed sequence of reals. Then the inequality

$$
\left\|\left(\sum_{x \in Q}\left|s_{Q}\right|^{q} c_{Q}^{q}\right)^{1 / q}\right\|_{L^{p}(d \omega)} \geq C\|s\|_{l^{r}}
$$

holds for all $s=\left\{s_{Q}\right\}$ if and only if one of the following conditions holds.

(a) $\max (p, q) \leq r<\infty$, and

$$
\inf _{Q} c_{Q}|Q|_{\omega}^{1 / p}>0 .
$$

(b) $p \leq r \leq q \leq \infty$, and for all dyadic cubes $P$,

$$
\sum_{Q \subset P}\left(c_{Q}^{q}|Q|_{\omega}\right)^{-r /(q-r)} \leq C|P|_{\omega}^{r(q-p) / p(q-r)} .
$$

(c) $0<r<\min (p, q)$, and

$$
\int\left[\sum_{x \in Q}\left(c_{Q}^{r}|Q|_{\omega}\right)^{-q /(q-r)}\right]^{p(q-r) / q(p-r)} d \omega<\infty
$$


(d) $q \leq r<p$, and

$$
\int \sup _{x \in Q}\left(c_{Q}^{r}|Q|_{\omega}\right)^{-p /(p-r)} d \omega<\infty
$$

Remark 5. We observe that, as in the case of Theorem 3 (see Remark 4), it follows from the proof presented below that the best constant in (3.15) satisfies the inequality $A_{1} \kappa(c) \leq C \leq A_{2} \kappa(c)$, where $A_{1}, A_{2}$ are positive constants which do not depend on $c=\left\{c_{Q}\right\}$, and $\kappa(c)$ is defined in terms of the quantities (3.16)-(3.19): $\kappa(c)=\inf _{Q} c_{Q}|Q|_{\omega}^{1 / p}$ if $\max (p, q) \leq r ; \kappa(c)=$ $C^{-(q-r) / q r}$ if $p \leq r \leq q$, where $C$ is the best constant in (3.17);

$$
\kappa(c)=\left\|\left(\sum_{x \in Q}\left(c_{Q}|Q|_{\omega}^{1 / r}\right)^{-q r /(q-r)}\right)^{(q-r) / q r}\right\|_{L^{p r /(p-r)}(d \omega)}^{-1}
$$

if $0<r<\min (p, q)$; and

$$
\kappa(c)=\left\|\sup _{x \in Q}\left(c_{Q}|Q|_{\omega}^{1 / r}\right)^{-1}\right\|_{L^{p r /(p-r)}(d \omega)}^{-1},
$$

if $q \leq r<p$.

Proof. Clearly $(3.15) \Rightarrow(3.16)$ for all $p, r, q$. Hence we may assume $c_{Q} \neq 0$ and $|Q|_{\omega} \neq 0$ for all dyadic cubes $Q$. If $\max (p, q) \leq r$, then by Lemma 2 (b) it follows that conversely, (3.16) $\Rightarrow(3.15)$, which proves statement (a).

Let us prove (b). Suppose $p \leq r \leq q$, and (3.17) holds. We choose $0<t<p$ and set $p_{1}=p /(p-t), q_{1}=q /(q-t), r_{1}=r /(r-t)$. Let $\phi_{Q}(x)=c_{Q} \chi(x)$ and $\psi_{Q}(x)=\phi_{Q}^{p-t}(x) /\left\|\phi_{Q}\right\|_{L^{p}(d \omega)}^{p}$. Then it is easily seen that $\psi_{Q}(x)=c_{Q}^{-t}|Q|_{\omega}^{-1} \chi_{Q}(x)$. By Corollary 1 , (3.15) holds if

$$
\left\|\left(\sum_{x \in Q}\left|s_{Q}\right|^{q_{1}} \psi_{Q}^{q_{1}}\right)^{1 / q_{1}}\right\|_{L^{p_{1}(d \omega)}} \leq C\|s\|_{l^{r_{1}}}
$$

By Theorem 3(b), the preceding inequality holds if, for any dyadic cube $P$,

$$
\sum_{Q \subset P}\left[\left(c_{Q}^{-t}|Q|_{\omega}^{-1}\right)^{q_{1}}|Q|_{\omega}\right]^{r_{1} /\left(r_{1}-q_{1}\right)} \leq C|P|_{\omega}^{r_{1}\left(p_{1}-q_{1}\right) / p_{1}\left(r_{1}-q_{1}\right)} .
$$

Since $r_{1}\left(p_{1}-q_{1}\right) / p_{1}\left(r_{1}-q_{1}\right)=r(q-p) / p(q-r)$ and $t q_{1} r_{1} /\left(r_{1}-q_{1}\right)=$ $q r /(q-r)$, it follows that the preceding condition is independent of $t$ and in fact coincides with (3.17). Thus (3.17) $\Rightarrow(3.15)$. 
The proof of the converse is similar to that given in [7, p. 77]. For any dyadic cube $P$ and integer $N \quad\left(l(P)>2^{-N}\right)$, we set $s=\left\{s_{Q}\right\}$, where $s_{Q}=\left(c_{Q}^{q}|Q|_{\omega}\right)^{-1 /(q-r)}$ if $Q \subset P$ and $l(Q) \geq 2^{-N}$, and $s_{Q}=0$ otherwise. Then by (3.15) for all cubes $Q, l(Q) \geq 2^{-N}$, we have

$$
\begin{aligned}
& C\left[\sum_{Q \subset P}\left(c_{Q}^{q}|Q|_{\omega}\right)^{-r(q-r)}\right]^{1 / r} \\
& \left.=C\|s\|_{l^{r}} \leq\left\|\left(\sum_{Q \subset P}|s|_{Q}^{q} c_{Q}^{q} \chi_{Q}\right)^{1 / q}\right\|_{L^{p}(d \omega)}^{p / q}\right)^{1 / p} . \\
& =\left(\int_{P}\left[\sum_{Q \subset P}\left(c_{Q}^{q}|Q|_{\omega}\right)^{-q /(q-r)} c_{Q}^{q} \chi_{Q}\right]^{p} .\right.
\end{aligned}
$$

By Hölder's inequality, the right-hand side of the preceding inequality is bounded by

$$
\begin{aligned}
& |P|_{\omega}^{1 / p-1 / q}\left(\int_{P} \sum_{Q \subset P}\left(c_{Q}^{q}|Q|_{\omega}\right)^{-q /(q-r)} c_{Q}^{q} \chi_{Q} d \omega\right)^{1 / q} \\
& =|P|_{\omega}^{1 / p-1 / q}\left[\sum_{Q \subset P}\left(c_{Q}^{q}|Q|_{\omega}\right)^{-r(q-r)}\right]^{1 / q} .
\end{aligned}
$$

Thus

$$
C\left[\sum_{Q \subset P}\left(c_{Q}^{q}|Q|_{\omega}\right)^{-r(q-r)}\right]^{1 / r} \leq|P|_{\omega}^{1 / p-1 / q}\left[\sum_{Q \subset P}\left(c_{Q}^{q}|Q|_{\omega}\right)^{-r(q-r)}\right]^{1 / q} .
$$

Since the sums are taken over the cubes $Q$ such that $l(Q) \geq 2^{-N}$, the righthand side of the preceding inequality is finite, and we get

$$
\sum_{Q \subset P}\left(c_{Q}^{q}|Q|_{\omega}\right)^{-r /(q-r)} \leq C|P|_{\omega}^{r(q-p) / p(q-r)} .
$$

Letting $N \rightarrow \infty$, we obtain (3.17). The proof of (b) is complete.

Now we prove statement (c). By Theorem 1 (ii) we get that $(3.18) \Rightarrow$ (3.15), as in the proof of Theorem 3 (c). To prove the converse, note that if (3.15) holds, then by Lemma 3 there exists $g=\left\{g_{Q}\right\}$ such that $\|g\|_{L^{p_{1}\left(l^{q_{1}}\right)}} \leq$ 1 , where $p_{1}=p /(p-r), q_{1}=q /(q-r)$, and

$$
\int\left|g_{Q}\right| \phi_{Q}^{r} d \omega=c_{Q}^{r} \int_{Q}\left|g_{Q}\right| d \omega \geq C,
$$


where $C$ does not depend on $Q$. From (3.20) it follows that

$$
\begin{aligned}
\int\left[\sum_{x \in Q}\left(c_{Q}^{r}|Q|_{\omega}\right)^{-q_{1}}\right]^{p_{1} / q_{1}} d \omega & \leq C \int\left[\sum_{x \in Q}\left(\frac{1}{|Q|_{\omega}} \int_{Q}\left|g_{Q}\right| d \omega\right)^{q_{1}}\right]^{p_{1} / q_{1}} d \omega \\
& \leq C\left\|M_{\omega}^{d} g\right\|_{L^{p_{1}\left(l^{q_{1}}\right)}}^{p_{1}} \leq C\|g\|_{L^{p_{1}\left(l^{q_{1}}\right)}}^{p_{1}} \leq C .
\end{aligned}
$$

Here $M_{\omega}^{d}$ is the vector-valued dyadic maximal operator. (See (3.7). Note that in the last inequality we used Theorem C. This part of the proof is similar to the argument in $[7$, pp. 78-79].) Thus (3.15) $\Rightarrow(3.18)$, which completes the proof of (c).

Now we prove statement (d). As in the proof of (c), it follows from Theorem 1 (ii) that $(3.19) \Rightarrow(3.15)$. We prove the converse first for $q=r$. Suppose (3.15) holds. Then by Lemma 3 (see Remark 2) there exists $F \in$ $L^{1}(d \omega), F \geq 0$, such that

$$
\inf _{Q} \int F^{1-r / p} \phi_{Q}^{r} d \omega=\inf _{Q} c_{Q}^{r} \int_{Q} F^{1-r / p} d \omega>0
$$

It follows from the preceding inequality

$$
\begin{aligned}
\int \sup _{x \in Q}\left(c_{Q}^{r}|Q|_{\omega}\right)^{-p /(p-r)} d \omega & \leq \int \sup _{x \in Q}\left(\frac{1}{|Q|_{\omega}} \int_{Q} F^{1-r / p} d \omega\right)^{p /(p-r)} d \omega \\
& \leq \int\left(M_{\omega}^{d} F^{1-r / p}\right)^{p /(p-r)} d \omega \leq C \int F d \omega<\infty .
\end{aligned}
$$

In the last inequality we used the fact that the maximal dyadic operator $M_{\omega}^{d}$ defined by (3.7) is bounded on $L^{p /(p-r)}(d \omega)$ (see [19]). Thus (3.19) holds.

It is more difficult to show that $(3.15) \Rightarrow(3.19)$ for $q<r$. Note that by duality

$$
\|s\|_{l^{r}}=\sup _{t=\left\{t_{Q}\right\}} \frac{\left(\sum\left|s_{Q}\right|^{q}\left|t_{Q}\right|^{q}\right)^{1 / q}}{\|t\|_{l^{r q /(r-q)}}} .
$$

Hence (3.15) is equivalent to the inequality

$$
\left(\sum\left|s_{Q}\right|^{q}\left|t_{Q}\right|^{q}\right)^{1 / q} \leq C\left\|\left(\sum_{x \in Q}\left|s_{Q}\right|^{q} c_{Q}^{q}\right)^{1 / q}\right\|_{L^{p}(d \omega)}\|t\|_{l^{r q /(r-q)}} .
$$

Letting $u=\left\{u_{Q}\right\}, v=\left\{v_{Q}\right\}$, where $u_{Q}=s_{Q} t_{Q}$ and $v_{Q}=c_{Q} /\left|t_{Q}\right|$, we rewrite the preceding estimate in the following form

$$
\|u\|_{l^{q}} \leq C\left\|\left(\sum_{x \in Q}\left|u_{Q}\right|^{q} v_{Q}^{q}\right)^{1 / q}\right\|_{L^{p}(d \omega)}\|t\|_{l^{r q /(r-q)}} .
$$


It follows from the argument given above for $q=r$ that

$$
\begin{aligned}
& \sup _{u=\left\{u_{Q}\right\}} \frac{\|u\|_{l^{q}}}{\|\left(\sum_{x \in Q}\left|u_{Q}\right|^{q} v_{Q}^{q}\right)^{1 / q}} \|_{L^{p}(d \omega)} \\
& \approx\left(\int \sup _{x \in Q}\left(v_{Q}^{q}|Q|_{\omega}\right)^{-p /(p-q)} d \omega\right)^{(p-q) /(p q)} .
\end{aligned}
$$

Since $v_{Q}=c_{Q} /\left|t_{Q}\right|$, from this we obtain

$$
\left(\int \sup _{x \in Q}\left(|t|_{Q}^{q} c_{Q}^{-q}|Q|_{\omega}^{-1}\right)^{p /(p-q)} d \omega\right)^{(p-q) /(p q)} \leq C\|t\|_{l^{r q /(r-q)}}
$$

for all $t=\left\{t_{Q}\right\}$. Let $p_{1}=p q /(p-q), r_{1}=r q /(r-q)$, and $\tilde{c}_{Q}=c_{Q}^{-1}|Q|_{\omega}^{-1 / q}$. Then (3.22) may be rewritten in the form

$$
\left(\int \sup _{x \in Q}\left(\widetilde{c}_{Q} t_{Q}\right)^{p_{1}} d \omega\right)^{1 / p_{1}} \leq C\|t\|_{l^{r_{1}}}
$$

Using the fact that $p_{1} r_{1} /\left(r_{1}-p_{1}\right)=p r /(p-r)$, and applying Theorem $3(\mathrm{~d})$, we get from the preceding inequality

$$
\int \sup _{x \in Q}\left(\widetilde{c}_{Q}^{r_{1}}|Q|_{\omega}\right)^{p_{1} /\left(r_{1}-p_{1}\right)} d \omega=\int \sup _{x \in Q}\left(c_{Q}^{r}|Q|_{\omega}\right)^{-p /(p-r)} d \omega<\infty .
$$

Thus $(3.15) \Rightarrow(3.19)$ in the case $q<r<p$. The proof of Theorem 4 is complete.

The following properties of discrete Carleson measures are immediate from Theorem 4 (in the case $r=p=1$ and $q=\infty$ ) and the result of L. Dor mentioned above (see Remark 3).

Corollary 2. Let $\left\{c_{Q}\right\}_{Q \in \mathcal{Q}}\left(c_{Q} \geq 0\right)$ be a fixed sequence of reals and let $\omega$ be a locally finite measure on $\mathbf{R}^{n}$. Then the following statements are equivalent.

(a) The inequality

$$
\int \sup _{x \in Q}\left|s_{Q}\right| d \omega \geq C \sum\left|s_{Q}\right| c_{Q}
$$

holds for all scalar sequences $s=\left\{s_{Q}\right\}$.

(b) $\sum_{Q \subset P} c_{Q} \leq C|P|_{\omega}$ for all dyadic cubes $P$.

(c) There exists a family of pairwise disjoint sets $\left\{E_{Q}\right\}_{Q \in \mathcal{Q}}$ such that $E_{Q} \subset$ $Q$ and $c_{Q} \leq C\left|E_{Q}\right|_{\omega}$. 


\section{Remark 6.}

(i) Theorem 4 is equivalent to the duality theorems for $\mathbf{f}$ spaces proved in [7] in the case where $d \omega$ is Lebesgue measure. As was mentioned in the Introduction, the proof given in [7] for the identity $\left(\mathbf{f}_{p}^{\alpha q}\right)^{*}=\mathbf{f}_{p^{\prime}}^{-\alpha \infty}$, which is equivalent to Theorem 4 (d), is not correct for $0<q<1$ and $p>1$. (Note that the Hahn-Banach theorem used in that proof fails for the space $L^{p}\left(l^{q}\right)$ in the non-locally-convex case $0<q<1$; see [13].) Our proof of Theorem 4 is based on duality and Theorem 3.

(ii) In case (b), which contains a discrete analogue of the $H^{1}$-BMO duality theorem, our proof is based on the dyadic Carleson measure theorem, rather than on the "local maximal functions" used in [7].

(iii) In the case $d \omega=d x$ and $p<r$, it can be shown that (3.17) is equivalent to the simpler condition (3.16) (cf. [7]). For $p=r$, as well as for arbitrary measures $\omega$, it can be shown that (3.16) generally does not imply (3.17).

\section{Multipliers of $f$ spaces.}

Let $\omega$ and $\sigma$ be locally finite measures on $\mathbf{R}^{n}$. We say that the sequence of reals $c=\left\{c_{Q}\right\}$ is a multiplier for the couple of $\mathbf{f}$ spaces, $\mathbf{f}_{p}^{\alpha q}(\sigma)$ and $\mathbf{f}_{p_{1}}^{\alpha_{1} q_{1}}(\omega)$, if

$$
\left\|\left\{c_{Q} s_{Q}\right\}\right\|_{\mathbf{f}_{p_{1}}^{\alpha_{1} q_{1}}(\omega)} \leq C\left\|\left\{s_{Q}\right\}\right\|_{\mathbf{f}_{p}^{\alpha q}(\sigma)}
$$

for all $s=\left\{s_{Q}\right\} \in \mathbf{f}_{p}^{\alpha q}(\sigma)$. In other words, $c$ is a multiplier if the corresponding multiplier operator defined by $C\left\{s_{Q}\right\}=\left\{c_{Q} s_{Q}\right\}$ is bounded. In this case we write $c \in \operatorname{Mult}\left(\mathbf{f}_{p}^{\alpha q}(\sigma) \rightarrow \mathbf{f}_{p_{1}}^{\alpha_{1} q_{1}}(\omega)\right)$.

In this section we characterize multipliers of $\mathbf{f}$ spaces in the "diagonal" case $p=p_{1}$ and $q=q_{1}$ for arbitrary $\omega$ and $\sigma$. We show that the multiplier problem is equivalent to the two weight problem for a generalized dyadic maximal operator considered in [21].

We may assume without loss of generality that the multipliers are nonnegative $\left(c_{Q} \geq 0\right)$. It is easily seen that $\tilde{c}=\left\{\widetilde{c}_{Q}\right\} \in \operatorname{Mult}\left(\mathbf{f}_{p}^{\alpha q}(\sigma) \rightarrow \mathbf{f}_{p}^{\alpha q}(\omega)\right)$ if and only if the following inequality holds,

$$
\left\|\left(\sum_{x \in Q} c_{Q}^{q}\left|s_{Q}\right|^{q}\right)^{1 / q}\right\|_{L^{p}(d \omega)} \leq C\left\|\left(\sum_{x \in Q}\left|s_{Q}\right|^{q}\right)^{1 / q}\right\|_{L^{p}(d \sigma)},
$$

where $c_{Q}=\tilde{c}_{Q}|Q|_{\sigma}^{1 / 2}|Q|_{\omega}^{-1 / 2}$. Thus the following theorem yields a characterization of the class of multipliers Mult $\left(\mathbf{f}_{p}^{\alpha q}(\sigma) \rightarrow \mathbf{f}_{p}^{\alpha q}(\omega)\right)$. 
Theorem 5. Let $0<p<\infty, 0<q \leq \infty$, and $c=\left\{c_{Q}\right\}, c_{Q} \geq 0$. Then (4.2) holds if and only if one of the following conditions is valid.

(a) $p=q$ and

$$
\sup _{Q} c_{Q} \frac{|Q|_{\omega}^{1 / p}}{|Q|_{\sigma}^{1 / p}}<\infty
$$

(b) $0<q<p$ and

$$
\int_{P} \sup _{x \in Q, Q \subset P}\left(c_{Q} \frac{|Q|_{\omega}^{1 / q}}{|Q|_{\sigma}^{1 / q}}\right)^{p q /(p-q)} d \sigma \leq C|P|_{\omega}
$$

for all dyadic cubes $P$.

(c) $0<p<q \leq \infty$ and

$$
\int_{P} \sup _{x \in Q, Q \subset P}\left(c_{Q} \frac{|Q|_{\omega}^{1 / q}}{|Q|_{\sigma}^{1 / q}}\right)^{p q /(q-p)} d \omega \leq C|P|_{\sigma}
$$

for all dyadic cubes $P$.

Proof. Note that (4.3) is clearly necessary in order that (4.2) hold for all $p$ and $q$. If $p=q$, then it is easily seen that the converse is also true since in this case $\mathbf{f}$ spaces turn into usual weighted $l^{p}$ spaces. This proves (a).

Suppose $q<p$. Then from Theorem 4 (c) with $q=\infty$ (see Remark 5) it follows

$$
\left\|\left(\sum_{x \in Q} c_{Q}^{q}\left|s_{Q}\right|^{q}\right)^{1 / q}\right\|_{L^{p}(d \omega)} \asymp \sup _{\left\{t_{Q}\right\}} \frac{\left(\sum_{Q} c_{Q}^{q}|Q| \omega\left|s_{Q}\right|^{q}\left|t_{Q}\right|^{q}\right)^{1 / q}}{\left\|\sup _{x \in Q}\left|t_{Q}\right|\right\|_{L^{p q /(p-q)}(d \omega)}} .
$$

Hence (4.2) holds if and only if

$$
\begin{aligned}
& \left(\sum_{Q} c_{Q}^{q}|Q|_{\omega}\left|s_{Q}\right|^{q}\left|t_{Q}\right|^{q}\right)^{1 / q} \\
& \leq C\left\|\sup _{x \in Q}\left|t_{Q}\right|\right\|_{L^{p q /(p-q)}(d \omega)}\left\|\left(\sum_{x \in Q}\left|s_{Q}\right|^{q}\right)^{1 / q}\right\|_{L^{p}(d \sigma)} .
\end{aligned}
$$

Applying Theorem 4 (d) with $r=q$, we get

$$
\sup _{\left\{s_{Q}\right\}} \frac{\left(\sum_{Q} c_{Q}^{q}|Q|_{\omega}\left|s_{Q}\right|^{q}\left|t_{Q}\right|^{q}\right)^{1 / q}}{\left\|\left(\sum_{x \in Q}\left|s_{Q}\right|^{q}\right)^{1 / q}\right\|_{L^{p}(d \sigma)}} \asymp\left\|\sup _{x \in Q}\left(c_{Q}\left|t_{Q}\right| \frac{|Q|_{\omega}^{1 / q}}{|Q|_{\sigma}^{1 / q}}\right)\right\|_{L^{p q /(p-q)}(d \sigma)} .
$$


Thus (4.2) is equivalent to the inequality

$$
\left\|\sup _{x \in Q}\left(c_{Q}\left|t_{Q}\right| \frac{|Q|_{\omega}^{1 / q}}{|Q|_{\sigma}^{1 / q}}\right)\right\|_{L^{p q /(p-q)}(d \sigma)} \leq C\left\|\sup _{x \in Q}\left|t_{Q}\right|\right\|_{L^{p q /(p-q)}(d \omega)}
$$

We define the generalized dyadic maximal operator (see [21]) by

$$
\mathcal{M} f(x)=\sup _{x \in Q}\left(\rho_{Q} \frac{1}{|Q|_{\omega}} \int_{Q}|f| d \omega\right)
$$

where the supremum is taken over all cubes $Q \in \mathcal{Q}_{0}=\left\{Q \in \mathcal{Q}:|Q|_{\omega} \neq 0\right\}$ containing $x$, and $\rho_{Q}$ are fixed non-negative reals associated with $Q \in \mathcal{Q}_{0}$.

Let $\rho_{Q}=c_{Q} \frac{|Q|_{\omega}^{1 / q}}{|Q|_{\sigma}^{1 / q}}$. We show that (4.6) is equivalent to the two weight maximal inequality

$$
\|\mathcal{M} f\|_{L^{p q /(p-q)}(d \sigma)} \leq C\|f\|_{L^{p q /(p-q)}(d \omega)} .
$$

By setting $f(x)=\sup _{x \in Q}\left|t_{Q}\right|$ in (4.7) and taking into account that

$$
\frac{1}{|Q|_{\omega}} \int_{Q}|f| d \omega \geq\left|t_{Q}\right|
$$

it is easily seen that $(4.7) \Rightarrow(4.6)$. To prove the converse, let

$$
t_{Q}=\frac{1}{|Q|_{\omega}} \int_{Q}|f| d \omega
$$

in (4.6) and use the fact that the dyadic maximal operator $M_{\omega}^{d}$ defined by (3.7) is bounded on $L^{p q /(p-q)}(d \omega)$. Hence (4.6) holds if and only if (4.7) is true.

It follows from the generalized two weight maximal inequality [21] that (4.7) holds if and only if

$$
\int_{P} \sup _{x \in Q, Q \subset P} \rho_{Q}^{p q /(p-q)} d \sigma \leq C|P|_{\omega}
$$

for all dyadic cubes $P$, which coincides with (4.4). This proves statement (b) of Theorem 5.

We use duality to show that (c) can be reduced to (b). Suppose $p<$ $q \leq \infty$. Using the transformation $\tilde{p}=p / t, \tilde{q}=q / t$, and $\widetilde{c}_{Q}=c_{Q}^{t}(t>0)$ if necessary, we see that, as in Sec. 2, we may assume without loss of generality $p>1$ and $q>1$. 
If $q=\infty$, then one can show that (4.2), which may be rewritten in the form

$$
\left\|\sup _{x \in Q} c_{Q} s_{Q}\right\|_{L^{p}(d \omega)} \leq C\left\|\sup _{x \in Q} s_{Q}\right\|_{L^{p}(d \sigma)}
$$

is equivalent to the generalized maximal inequality

$$
\left\|\sup _{x \in Q}\left(c_{Q} \frac{1}{|Q|_{\sigma}} \int_{Q}|f| d \sigma\right)\right\|_{L^{p}(d \omega)} \leq C\|f\|_{L^{p}(d \sigma)},
$$

where $f \in L^{p}(d \sigma)$. (See the proof of the equivalence of (4.6) and (4.7) above.) Then it follows from [21] again that the preceding maximal inequality is characterized by

$$
\int_{P} \sup _{x \in Q, Q \subset P} c_{Q}^{p} d \omega \leq C|P|_{\sigma}
$$

which coincides with (4.5) for $q=\infty$.

Suppose $1<p<q<\infty$. It follows from the duality theorems for $\mathbf{f}$ spaces (or, which is equivalent, from Theorem 4 (c)) that (4.2) holds if and only if

$$
\left\|\left[\sum_{x \in Q} c_{Q}^{q^{\prime}}\left(\frac{|Q|_{\omega}}{|Q|_{\sigma}}\right)^{q^{\prime}}\left|s_{Q}\right|^{q^{\prime}}\right]^{1 / q^{\prime}}\right\|_{L^{p^{\prime}}(d \sigma)} \leq C\left\|\left(\sum_{x \in Q}\left|s_{Q}\right|^{q^{\prime}}\right)^{1 / q^{\prime}}\right\|_{L^{p^{\prime}}(d \omega)}
$$

Since $p^{\prime}>q^{\prime}$, it is easily derived from (b) that (4.8) holds if and only if (4.5) is true. The proof of Theorem 5 is complete.

\section{References}

[1] D.R. Adams and L.I. Hedberg, Function Spaces and Potential Theory, SpringerVerlag, Berlin-Heidelberg-New York, 1996.

[2] E. Amar and A. Bonami, Mesures de Carleson d'order $\alpha$ et solutions au bord de l'equation $\bar{\partial}$, Bull. Soc. Math. France, 107 (1979), 124-153.

[3] L. Carleson, An interpolation problem for bounded analytic functions, Amer. J. Math., 80 (1958), 921-930.

[4] L. Dor, On projections in $L^{1}$, Ann. Math., 102 (1975), 463-474.

[5] C. Fefferman and E.M. Stein, Some maximal inequalities, Amer. J. Math., 93 (1971), 107-115.

[6] C. Fefferman and E.M. Stein, $H^{p}$ spaces of several variables, Acta Math., 129 (1972), 137-193.

[7] M. Frazier and B. Jawerth, A discrete transform and decompositions of distribution spaces, J. Funct. Analysis, 93 (1990), 34-170. 
[8] M. Frazier, B. Jawerth and G. Weiss, Littlewood-Paley Theory and the Study of Function Spaces, CBMS-AMS Regional Conf., 79 (1991).

[9] J. Garcia-Cuerva and J.-L. Rubio de Francia, Weighted Norm Inequalities and Related Topics, North-Holland Math. Studies, 116, North-Holland, Amsterdam (1985).

[10] J. Garnett, Bounded Analytic Functions, Acad. Press, New York-London-Toronto, 1981.

[11] Y.S. Han and E.T. Sawyer, Littlewood-Paley Theory on Spaces of Homogeneous Type and the Classical Function Spaces, Memoirs Amer. Math. Soc., 530 (1994).

[12] N.J. Kalton and S.J. Montgomery-Smith, Set-functions and factorization, Arch. Math., 61 (1993), 183-200.

[13] N.J. Kalton, N.T. Peck and J.W. Roberts, An F-Space Sampler, London Math. Soc. Lecture Notes, 89 (1984).

[14] D.H. Luecking, Embedding derivatives of Hardy spaces into Lebesgue spaces, Proc. London Math. Soc., 63 (1991), 595-619.

[15] _ Embedding theorems for spaces of analytic functions via Khinchine's inequality, Michigan Math. J., 40 (1993), 333-358.

[16] B. Maurey, Theórèmes de factorisation pour les opérateurs linéaires à valeurs dans les espaces $L^{p}$, Astérisque, 11 (1974), 1-163.

[17] Y. Meyer, Wavelets and operators, Analysis at Urbana, London Math. Soc. Lecture Notes Series, 137, 256-364, Cambridge Univ. Press, 1989.

[18] G. Pisier, Factorization of operators through $L^{p \infty}$ or $L^{p 1}$ and noncommutative generalizations, Math. Ann., 276 (1986), 105-136.

[19] E.T. Sawyer, A characterization of a two weight maximal norm inequality for maximal operators, Studia Math., 75 (1982), 1-11.

[20] E.T. Sawyer and R.L. Wheeden, Weighted inequalities for fractional integrals on Euclidean and homogeneous spaces, Amer. J. Math., 114 (1992), 813-874.

[21] I.E. Verbitsky, Weighted norm inequalities for maximal operators and Pisier's theorem on factorization through $L^{p \infty}$, Int. Equat. Oper. Theory, 15 (1992), 124-153.

[22] I.E. Verbitsky and R.L. Wheeden, Weighted inequalities for fractional integrals and applications to semilinear equations, J. Funct. Analysis, 129 (1995), 221-241.

Received January 26, 1995 and revised November 20, 1995. Supported in part by NSF Grant DMS94-01493.

University of Missouri - Columbia

Columbia, MO 65211

E-mail address:igor@fermat.math.missouri.edu 


\title{
PACIFIC JOURNAL OF MATHEMATICS
}

\author{
Founded in 1951 by
}

\author{
$\begin{array}{ll}\text { E. F. Beckenbach (1906-1982) } & \text { F. Wolf (1904-1989) }\end{array}$
}

\section{EDITORS}

Sun-Yung A. Chang (Managing Editor) Robert Finn University of California

Los Angeles, CA 90095-1555

pacific@math.ucla.edu

\section{F. Michael Christ}

University of California

Los Angeles, CA 90095-1555

christ@math.ucla.edu

Nicholas Ercolani

University of Arizona

Tucson, AZ 85721

ercolani@math.arizona.edu
Stanford University

Stanford, CA 94305

finn@gauss.stanford.edu

Steven Kerckhoff

Stanford University

Stanford, CA 94305

spk@gauss.stanford.edu

Martin Scharlemann

University of California

Santa Barbara, CA 93106

mgscharl@math.ucsb.edu

\section{Gang Tian}

Massachusettes Institute of Technology

Cambridge, MA 02139

tian@math.mit.edu

\section{S. Varadarajan University of California Los Angeles, CA 90095-1555 vsv@math.ucla.edu \\ Dan Voiculescu \\ University of California \\ Berkeley, CA 94720 \\ dvv@math.berkeley.edu}

\section{SUPPORTING INSTITUTIONS}

ACADEMIA SINICA, TAIPEI

CALIF. INST. OF TECHNOLOGY

CHINESE UNIV. OF HONG KONG

HONG KONG UNIV. OF SCI. \& TECH.

KEIO UNIVERSITY

MACQUARIE UNIVERSITY

MATH. SCI. RESEARCH INSTITUTE

NEW MEXICO STATE UNIV.

OREGON STATE UNIV.

PEKING UNIVERSITY

RITSUMEIKAN UNIVERSITY

STANFORD UNIVERSITY

\author{
TOKYO INSTITUTE OF TECHNOLOGY \\ UNIVERSIDAD DE LOS ANDES \\ UNIV. OF ARIZONA \\ UNIV. OF BRITISH COLUMBIA \\ UNIV. OF CALIF., BERKELEY \\ UNIV. OF CALIF., DAVIS \\ UNIV. OF CALIF., IRVINE \\ UNIV. OF CALIF., LOS ANGELES \\ UNIV. OF CALIF., RIVERSIDE \\ UNIV. OF CALIF., SAN DIEGO \\ UNIV. OF CALIF., SANTA BARBARA
}

UNIV. OF CALIF., SANTA CRUZ

UNIV. OF HAWAII

UNIV. OF MELBOURNE

UNIV. OF MONTANA

UNIV. NACIONAL AUTONOMA DE MEXICO

UNIV. OF NEVADA, RENO

UNIV. OF OREGON

UNIV. OF SOUTHERN CALIFORNIA

UNIV. OF UTAH

UNIV. OF WASHINGTON

WASHINGTON STATE UNIVERSITY

The supporting Institutions listed above contribute to the cost of publication of this Journal, but they are not owners or publishers and have no responsibility for its contents or policies.

Manuscripts must be prepared in accordance with the instructions provided on the inside back cover.

The table of contents and the abstracts of the papers in the current issue, as well as other information about the Pacific Journal of Mathematics, may be found on the Internet at http://www.math.uci.edu/pjm.html.

The Pacific Journal of Mathematics (ISSN 0030-8730) is published monthly except for July and August. Regular subscription rate: $\$ 245.00$ a year (10 issues). Special rate: $\$ 123.00$ a year to individual members of supporting institutions.

Subscriptions, back issues published within the last three years and changes of subscribers address should be sent to Pacific Journal of Mathematics, P.O. Box 4163, Berkeley, CA 94704-0163, U.S.A. Prior back issues are obtainable from Kraus Periodicals Co., Route 100, Millwood, NY 10546.

The Pacific Journal of Mathematics at the University of California, c/o Department of Mathematics, 981 Evans Hall, Berkeley, CA 94720 (ISSN 0030-8730) is published monthly except for July and August. Second-class postage paid at Berkeley, CA 94704, and additional mailing offices. POSTMASTER: send address changes to Pacific Journal of Mathematics, P.O. Box 6143, Berkeley, CA 94704-0163.

\section{PUBLISHED BY PACIFIC JOURNAL OF MATHEMATICS at University of California,} Berkeley, CA 94720, A NON-PROFIT CORPORATION

This publication was typeset using AMS-LATEX,

the American Mathematical Society's TEX macro system.

Copyright (C) 1995 by Pacific Journal of Mathematics 


\section{PACIFIC JOURNAL OF MATHEMATICS}

\section{Volume $176 \quad$ No. $2 \quad$ December 1996}

One remark on polynomials in two variables

ENRIQUe ARTAl BARTOLO and PIERRETTE CASSOU-NOGUÈS

Divergence of the normalization for real Lagrangian surfaces near complex tangents XIANGHONG GONG

Classification of the stable homotopy types of stunted lens spaces for an odd prime JESUS GONZALEZ

Plancherel formulae for non-symmetric polar homogeneous spaces

JING-SONG HUANG

A uniqueness theorem for the minimal surface equation

JENN-FANG HWANG

Differential Galois groups of confluent generalized hypergeometric equations: an approach

using Stokes multipliers

Claudine Mitschi

Oscillatory theorem and pendent liquid drops

KIMIAKI NARUKAWA and TAKASHI SUZUKI

Local and global plurisubharmonic defining functions

ALAN NOELL

Specializations and a local homeomorphism theorem for real Riemann surfaces of rings

M. J. DE LA PUENTE

Eigenvalue comparisons in graph theory

GREGORY T. QUENELL

Applications of loop groups and standard modules to Jacobians and theta functions of isospectral curves

WILLI SCHWARZ

Bridged extremal distance and maximal capacity

ROBERT E. THURMAN

Imbedding and multiplier theorems for discrete Littlewood-Paley spaces

IGOR E. VERBITSKY

On constrained extrema

THOMAS VOGEL

Heat flow of equivariant harmonic maps from $\mathbb{B}^{3}$ into $\mathbb{C P} \mathbb{P}^{2}$

YUANLONG XIN

Proof of Longuerre's theorem and its extensions by the method of polar coordinates

ZHIHONG YU

Correction to: "Special generating sets of purely inseparable extension fields of unbounded exponent"

BONIFACE IHEMOTUONYE EKE 